\title{
Modelo para el acceso efectivo al tratamiento antirretroviral en relación con el fracaso terapéutico de la infección por VIH
}

\author{
- HERnÁn DARÍo TORO-ZAPATA ${ }^{1}$ \\ ANGIE JOHANNA OSORIO-RODRÍGUEZ ${ }^{1}$ \\ DenNis AleXander Prieto-Medellin ${ }^{1}$
}

\section{Resumen}

Se plantea un modelo matemático con base en ecuaciones diferenciales ordinarias no lineales que interpreta el efecto que el acceso efectivo y el fracaso terapéutico tienen en una población infectada con el VIH, en la que se considera únicamente la transmisión del virus es por vía sexual con una persona portadora. Se hace el análisis del modelo matemático, determinando el número básico de reproducción $R_{0}$, los puntos de equilibrio del sistema y se prosigue estableciendo la estabilidad local de los mismos. Posteriormente se determinan dos umbrales que sugieren condiciones para evitar la manifestación de la infección en la población. El primer umbral relaciona el fracaso terapéutico en función del acceso efectivo al tratamiento antirretroviral y el segundo umbral es solo para el acceso efectivo al tratamiento en función de otros parámetros del modelo; en cada caso se determinan cuáles son las cantidades mínimas que se deben asegurar tanto de acceso efectivo como de fracaso terapéutico para garantizar teóricamente que la enfermedad sea controlada.

Palabras claves: Sistema dinámico; Tratamiento antirretroviral; Acceso efectivo; Fracaso terapéutico.

\section{Model for effective access to antiretroviral treatment in relation to the therapeutic failure of HIV infection}

\section{Abstract}

A mathematical model based on ordinary nonlinear differential equations is proposed to interpret the effect of effective access and therapeutic failure in HIV infection among a population. The model only considers sexual

1 Universidad del Quindío, Armenia, Colombia.

Autor de correspondencia: Toro-Zapata, H.D. (Hernán Darío): Universidad del Quindío, Carrera 15 Calle 12 Norte, Armenia, Quindío, Colombia. Teléfono: +57 (6) 7359300. Correo electrónico: hdtoro@uniquindio.edu.co
Historia del artículo:

Artículo recibido: 2-IV-2018/ Aprobado: 20-V-2018

Disponible online: 3 de febrero de 2019

Discusión abierta hasta octubre de 2020 
transmission. The analysis is made by determining the basic reproduction number, $R_{0}$, the equilibrium points of the system and their local stability. Subsequently, two thresholds are determined to stablish sufficient conditions to avoid the manifestation of infection among the population. The first threshold is relates to therapeutic failure and based on access to antiretroviral treatment; the second threshold is only related to access to treatment and other parameters of the model; in each case they are determined to be the minimum quantities that must hold to have effective access and therapeutic failure, and therefore, to guarantee smaller levels of infection.

Keywords: Dynamic system; Antiretroviral treatment; Effective access; Therapeutic failure.

\section{Modelo para acesso efetivo ao tratamento anti-retroviral em relação à falha terapêutica da infecção pelo HIV}

\section{Resumo}

Um modelo matemático é proposto e baseado em equações diferenciais não lineares que interpretam o efeito que o acesso eficaz e o fracasso do tratamento ocorre numa população infectada pelo HIV em que só a transmissão do vírus é considerada sexualmente transmitida com uma pessoa portadora. É analisado o método matemático, determinando o número básico de reprodução $R_{0}$, os pontos de equilíbrio do sistema e seguidamente é estabelecida a estabilidade local dos mesmos. Posteriormente, são determinados dois limiares que sugerem condições para evitar a manifestação da infecção na população. 0 primeiro limiar refere-se ao fracasso do tratamento com base no acesso eficaz para a terapia anti-retroviral e o segundo limiar é o único acesso ao tratamento eficaz fundamentado em outros parâmetros do modelo. Em cada caso, são determinadas as quantias mínimas que devem ser mantidas tanto para o acesso efetivo ao tratamento como para a falha terapêutica, garantindo teoricamente que a doença é controlada.

Palavras-chave: Sistema dinâmico; Terapia anti-retroviral; Acesso eficaz; Fracasso do tratamento.

\section{Introducción}

La infección por el Virus de Inmunodeficiencia Humana (VIH) y el Síndrome de Inmunodeficiencia Adquirida (SIDA) son un problema de salud a escala mundial, que tiene impactos negativos a nivel psicológico, biológico, sociocultural y económico, tanto en la persona infectada como en sus familiares (Organización Mundial de la Salud, 2015). Las principales vías de transmisión del virus del VIH son: el contagio por relaciones sexuales con una persona portadora del virus, por transfusión de sangre contaminada, por compartir agujas, jeringas o diferentes objetos punzocortantes o de la madre al feto (Grupo Traba- jo sobre Tratamientos del VIH, 2011)El virus logra desgastar el sistema inmune hasta que produce una deficiencia que incrementa el riesgo de sufrir infecciones oportunistas. Una vez la persona es diagnosticada como portadora del VIH, deberá iniciar tratamiento antirretroviral (TAR), que generalmente consiste en un politerapia constituida por tres o más antirretrovirales, los cuales actúan inhibiendo la replicación del virus, bloqueando las enzimas del VIH o creando nuevas barreras para la enfermedad.

El concepto de acceso efectivo al TAR hace referencia a la proporción de personas que pueden iniciar el uso de antirretrovirales. Generalmente, dado el alto costo de los tratamientos frente al VIH, 
el acceso es limitado y depende mucho de su inclusión en los sistemas de salud de los países. Se debe tener en cuenta que el concepto de los altos costos afecta directamente a la calidad de medicamentos utilizados, dado que aunque los medicamentos de primera línea generan mejores resultados disminuyendo los efectos secundarios y la resistencia del virus a los medicamentos, producen mayores costos, y es por esto que generalmente en los países del sur global se utilizan medicamentos de segunda línea que brindan resultados aceptables (Zaidi, 2011).

En Colombia, se rige por la resolución número 5592 del 24 de diciembre de 2015 del Ministerio de Salud y Protección Social, en la cual se establece todo lo relacionado con el Plan de Beneficios en Salud con cargo a la Unidad de Pago por Capitación (UPS) del Sistema General de Seguridad Social en Salud (SGSSS) (Ministerio de Salud y Protección Socia, 2015).

Es fundamental la capacidad del paciente para seguir las indicaciones del médico de manera adecuada, concepto que se denomina adherencia y se refiere a todo lo relacionado con el correcto cumplimiento del tratamiento antirretroviral (Tarinas, et al., 2000), la adherencia presenta dos tipos de barreras, adherencia farmacológica y adherencia no farmacológica, relacionadas con factores como: olvido, múltiples ocupaciones, el estar enfermo o deprimido, experimentar efectos secundarios a causa del uso de los antirretrovirales, el tipo de alimentación, la actividad física, el consumo de bebidas alcohólicas y sustancias psicoactivas, e interacciones medicamentosas (infosida, 2005), (infosida, 2017), (Santillán, 2014), (Varela \& Hoyos, 2015).

En el ámbito del modelado del VIH y SIDA, se encuentran diversas publicaciones donde proponen modelos matemáticos en los que se contempla la dinámica del virus a nivel inmunológico o la dinámica poblacional de la enfermedad. Algunos modelos planteados en la literatura se han enfocado en el modelado de la infección y el tratamiento (Adams, et al., 2005), (Campos \& Palacios, 2005), (Cleary, et al., 2010), (Culshaw, et al., 2004), (Del Valle, et al., 2004), (Ding \& Wu, 1999), (Hsu, 2000), (Nelson, et al., 2000), (Revilla \& García, 2003), (Shim, et al., 2003), (Stengel, 2008), (Wandeler, et al., 2011), (Zaric, et al., 2008); en el modelado de costos (Haacker, 2002), (Oliva, et al., 2010) , (Trujillo \& Toro, 2015), (Zaric, et al., 2008) el modelado de la dinámica del VIH (Dalal, et al., 2008), (Tan \& Byers, 1993), (Taylor, et al., 1994) o han hecho uso de los procesos estocásticos para modelar la dinámica (Chao, et al., 2004), (Dalal, et al., 2008), (Gray, et al., 2003) (Hsu, 2000), (Jiang, et al., 2017), (Kouyos, et al., 2006), (Ribeiro \& Bonhoeffer, 1999).

En particular, en lo que respecta al número básico de reproducción, Rivera B., et al. (2011), estudian la dinámica de VIH-SIDA en la ciudad de Cali por medio de un modelo SIR, toman como supuesto que la transmisión sexual es el único medio de contagio, estiman el número básico de reproducción y encuentran dos puntos de equilibrio (Sepulveda, et al., 2011).

Nelson P., et al. (2000), investigan las mediciones experimentales sobre el VIH, especialmente utilizando datos de experimentos en la administración de potentes fármacos antirretrovirales y considerando que tras la infección de una célula, esta comienza inmediatamente a producir el virus (Nelson, et al., 2000). Por otro lado, Marco A. (2012), observa una relación entre el cumplimiento del tratamiento antirretroviral con el fracaso virológico, llegando a una implicación directa del concepto de adherencia en las personas que tienen tratamiento antirretroviral (Marco, 2012).

En correspondencia con lo anterior, en la sección 2 se propone un modelo matemático basado en ecuaciones diferenciales ordinarias, que interprete la dinámica de infección por el virus del VIH en una población humana considerando individuos en un intervalo de edad de 15 a 25 años (sin consideraciones de género u orientación sexual), con el fin de estudiar el efecto que, el acceso efectivo y el fracaso terapéutico al TAR tienen sobre la dinámica de la enfermedad; en la sección 3 se plantea un problema de control óptimo, considerando un funcional de costos que acumule los costos directos e indirectos del acceso efectivo y el abandono al TAR, determinando un problema 
de contorno el cual es solucionado por medio de un software matemático, y por último en la sección 4 se establecen algunas conclusiones relacionadas con los umbrales propuestos en la sección 2 .

\section{Planteamiento del modelo}

En la formulación del modelo, se asume que la población humana se encuentra dividida en tres categorías o subpoblaciones, que corresponden a (i) personas susceptibles de adquirir el virus; es decir, personas que son sexualmente activas en un rango de edad de 15 a 25 años y que podrían, eventualmente, entrar en contacto con personas infectadas; (ii) personas infectadas con el virus pero que no están en TAR, bien sea porque no han sido diagnosticados, porque no han iniciado el tratamiento, o porque han decidido abandonarlo. Finalmente, (iii) personas infectadas que se encuentran en TAR.

Sea la variable de estado $x_{1}=x_{1}(t)$ que denota el número promedio de personas susceptibles a adquirir el virus del VIH en un determinado tiempo $t$, la variable de estado $x_{2}=x_{2}(t)$ que representa el número promedio de personas portadoras del virus $\sin$ TAR y $x_{3}=x_{3}(t)$ el número promedio de personas infectadas que acceden efectivamente al tratamiento. Se asume que la población $x_{1}$ crece a una tasa constante $\Delta$ y que disminuye proporcionalmente a su tamaño según una tasa de muerte natural $\mu$, de manera que $\mu x_{1}$ denota el número promedio de personas susceptibles que mueren por causas naturales. Se considera que $\beta_{1} x_{1} x_{2}$ representa el número promedio de contactos efectivos entre infectados sin tratamiento y susceptibles, y $\beta_{2} x_{1} x_{3}$ es el número promedio de contactos efectivos entre infectados con tratamiento y susceptibles; lo que implica asumir que la tasa de infección puede ser descrita por la ley de acción de masas, formulada por Hamer en 1906 y la cual describe que el número de contactos infecciosos, por unidad de tiempo es proporcional al número total de contactos entre individuos infectados y sanos (Cuddington \& Beisner, 2005). Según lo anterior, la variación de la población susceptible con respecto al tiempo se describe por la ecuación:

$$
\frac{d x_{1}}{d t}=\Delta-\beta_{1} x_{1} x_{2}-\beta_{2} x_{1} x_{3}-\mu x_{1}
$$

Sea $\alpha x_{2}$ el término que representa al número promedio de personas infectadas que "acceden efectivamente" al tratamiento antirretroviral, por lo tanto $\alpha$ denota la proporción de acceso efectivo a tratamiento; es decir, $\alpha=0$ implica que ninguna persona infectada puede acceder al tratamiento, mientras que $\alpha=1$ implica que el $100 \%$ de la población infectada accede efectivamente al tratamiento, es decir, cobertura completa. Considere ahora que las personas infectadas sin tratamiento, tienen una tasa de muerte asociada con la enfermedad $\delta$, así que $\delta x_{2}$ denota el número promedio de personas que mueren como consecuencia de la infección, en general, estas muertes están asociadas con la aparición de enfermedades oportunistas. Nuevamente, $\operatorname{con} \mu x_{2}$ se denota el número promedio de personas infectadas sin tratamiento que mueren por causas naturales. La ecuación que describe la variación de $x_{2}$ con respecto al tiempo, queda de la forma:

$$
\frac{d x_{2}}{d t}=\beta_{1} x_{1} x_{2}+\beta_{2} x_{1} x_{3}+\gamma x_{3}-(\delta+\alpha+\mu) x_{2}
$$

Finalmente, se asume que la variación de la población infectada con tratamiento $x_{3}$ está dada por:

$$
\frac{d x_{3}}{d t}=\alpha x_{2}-(\mu+\phi+\gamma) x_{3}
$$

donde $\mu x_{3}$ denota el número promedio de personas infectadas y con TAR que mueren por causas naturales. También considera que las personas infectadas con tratamiento, tienen una tasa de muerte asociada con la enfermedad $\phi$ así $\phi x_{3}$ denota el número promedio de personas infectadas sin tratamiento que mueren como consecuencia de la infección, $\gamma x_{3}$ denota la proporción de personas que presenta fracaso terapéutico, por lo cual se está asumiendo que $1-\gamma$ es la proporción de personas que se "adhieren" al tratamiento, y $\gamma$ es la proporción de personas infectadas que lo abandonan, es decir, tuvieron fracaso terapéutico. 
El sistema de ecuaciones diferenciales ordinarias y no lineales que interpreta la dinámica del proceso es:

$$
\begin{aligned}
& \frac{d x_{1}}{d t}=\Delta-\beta_{1} x_{1} x_{2}-\beta_{2} x_{1} x_{3}-\mu x_{1} \\
& \frac{d x_{2}}{d t}=\beta_{1} x_{1} x_{2}+\beta_{2} x_{1} x_{3}+\gamma x_{3}-(\delta+\alpha+\mu) x_{2} \\
& \frac{d x_{3}}{d t}=\alpha x_{2}-(\mu+\phi+\gamma) x_{3}
\end{aligned}
$$

Donde $\Delta, \mu, \delta, \phi>0,0 \leq \beta_{1} \leq 1,0 \leq \beta_{2} \leq 1,0 \leq \alpha$ $\leq 1,0 \leq \gamma \leq 1$ y las condiciones iniciales son $x_{1}(0)=$ $x_{10}, x_{2}(0)=x_{20} y x_{3}(0)=x_{30}$ las cuales se asumen no negativas. La descripción de variables y parámetros pueden verse en el Cuadro 1.

Proposición 1: Las soluciones del sistema (4), $\Omega=\left\{\left(x_{1}, x_{2}, x_{3}\right) \in \mathbb{R}_{+}^{3}: 0 \leq x_{1}+x_{2}+x_{3} \leq \frac{\Delta}{\mu}\right\}$ es un conjunto positivamente invariante:

$$
\Omega=\left\{\left(x_{1}, x_{2}, x_{3}\right) \in \mathbb{R}_{+}^{3}: 0 \leq x_{1}+x_{2}+x_{3} \leq \frac{\Delta}{\mu}\right\}
$$

Prueba: Para que las soluciones sean no negativas; note que,

$$
\left.\frac{d x_{1}}{d t}\right|_{x_{1}=0}=\Delta>0
$$

Por lo tanto $x_{1}(t)$ es creciente, luego la función $x_{1}(t)$ será no negativa. De manera semejante,

$$
\left.\frac{d x_{2}}{d t}\right|_{x_{2}=0}=\beta_{2} x_{1} x_{3}+\gamma x_{3}
$$

Como $\gamma \in[0,1]$ se obtiene $\beta_{2} x_{1} x_{3}+\gamma x_{3} \geq 0$, en cuyo caso se concluye que $x_{2}(t)$ es creciente y no negativa. Por último,

$$
\left.\frac{d x_{3}}{d t}\right|_{x_{3}=0}=\alpha x_{2}
$$

De donde se concluye que $\alpha x_{2} \geq 0$ debido a que $\alpha>0$, por lo tanto $x_{3}(t)$ es creciente y no negativa. Para probar que las soluciones están acotadas superiormente, sea $N=x_{1}+x_{2}+x_{3}$, por lo tanto su variación respecto a $t$ está dada por:

$$
\frac{d N}{d t}=\Delta-\mu N-\delta x_{2}-\phi x_{3} \leq \Delta-\mu N
$$

Por lo tanto $N(t) \leq \frac{\Delta}{\mu}\left(1-e^{-\mu t}\right)$ de donde $N(t) \leq \frac{\Delta}{\mu}$ cuando $t \rightarrow \infty$, por lo anterior se concluye que la región de $\mathbb{R}^{3}$ definida por:

$$
\Omega=\left\{\left(x_{1}, x_{2}, x_{3}\right) \in \mathbb{R}_{+}^{3}: 0 \leq x_{1}+x_{2}+x_{3} \leq \frac{\Delta}{\mu}\right\}
$$

es un conjunto positivamente invariante para sistema (4).

\subsection{Análisis del modelo}

Para el modelo formulado anteriormente se hace un análisis que consiste en determinar el número básico de reproducción $R_{0}$, se encuentran los puntos de equilibrios que presenta el modelo y la estabilidad local de los mismos, se finaliza determinado dos umbrales de control con base en el nivel de acceso efectivo y del fracaso terapéutico, finalmente se muestran las simulaciones del sistema de ecuaciones diferenciales.

\subsubsection{Número básíco de reproducción $\boldsymbol{R}_{0}$}

El número básico de reproducción $R_{0}$, está definido como el número esperado de casos secundarios producidos por un individuo infectado típico durante todo su período de infecciosidad en una población susceptible demográficamente estable, matemáticamente se puede definir como el radio espectral de la matriz de la siguiente generación (Heesterbeek, 2002). Para el sistema (4) el $R_{0}$ está dado por:

$$
\begin{gathered}
R_{0}=\frac{\beta_{1} \Delta}{(\delta+\alpha+\mu) \mu}+\frac{\beta_{2} \Delta \alpha}{\mu(\delta+\alpha+\mu)(\gamma+\mu+\phi)}+ \\
\frac{\gamma \alpha}{(\delta+\alpha+\mu)(\gamma+\mu+\phi)}
\end{gathered}
$$


TABLA 1. DESCRIPCIÓN DE LAS VARIABLES Y PARÁMETROS USADOS EN EL MODELO (4), LOS VALORES ASIGNADOS PARA SIMULACIÓN Y LA FUENTE BIBLIOGRÁFICA DE DONDE SON OBTENIDOS

\begin{tabular}{|c|c|c|c|}
\hline Símbolo & Descripción & Valor & Referencias \\
\hline$x_{1}(t)$ & $\begin{array}{l}\text { Número promedio de personas susceptibles en un } \\
\text { tiempo } t \text {. }\end{array}$ & 1000 & - \\
\hline$x_{2}(t)$ & $\begin{array}{l}\text { Número promedio de personas infectadas con VIH } \\
\text { sin tratamiento en un tiempo } t \text {. }\end{array}$ & 1 & - \\
\hline$x_{3}(t)$ & $\begin{array}{l}\text { Número promedio de personas infectadas con VIH } \\
\text { con tratamiento en un tiempo } t \text {. }\end{array}$ & 0 & - \\
\hline$\Delta$ & $\begin{array}{l}\text { Tasa de incremento constante de las personas } \\
\text { susceptibles. }\end{array}$ & 10 & (Mesa, et al., 2014) \\
\hline$\beta_{1}$ & $\begin{array}{l}\text { Tasa de contactos efectivos entre susceptibles y } \\
\text { personas infectadas sin tratamiento antirretroviral. }\end{array}$ & 0.00042 & (Mesa, et al., 2014) \\
\hline$\beta_{2}$ & $\begin{array}{l}\text { Tasa de contactos efectivos entre susceptibles y } \\
\text { personas infectadas con tratamiento antirretroviral. }\end{array}$ & 0.00001 & (Mesa, et al., 2014) \\
\hline$\alpha$ & $\begin{array}{l}\text { Proporción de acceso de infectados a tratamiento } \\
\text { antirretroviral. }\end{array}$ & Variable & \\
\hline$\gamma$ & $\begin{array}{l}\text { Proporción de personas que abandonan el } \\
\text { tratamiento antirretroviral. }\end{array}$ & Variable & - \\
\hline$\mu$ & Tasa de mortalidad por causas ajenas a la infección. & 0.01 & (Mesa, et al., 2014) \\
\hline$\delta$ & $\begin{array}{l}\text { Tasa de mortalidad de las personas con VIH sin } \\
\text { vinculación al tratamiento por causas relacionadas } \\
\text { con la enfermedad. }\end{array}$ & 0.02 & $\begin{array}{l}\text { (Instituto Nacional de Salud, } \\
\text { 2016) }\end{array}$ \\
\hline$\phi$ & $\begin{array}{l}\text { Tasa de mortalidad de las personas con VIH } \\
\text { vinculados al tratamiento por causas relacionadas } \\
\text { con la enfermedad. }\end{array}$ & 0.01 & (Mesa, et al., 2014) \\
\hline
\end{tabular}

Para una mejor comprensión del significado del número básico de reproducción $R_{0}$ se debe tener en cuenta que se presentan efectos aditivos y multiplicativos, el término $\frac{\beta_{1} \Delta}{(\delta+\alpha+\mu) \mu}$ representa los nuevos casos que produce una persona infectada sin tratamiento antirretroviral los cuales se encuentran en la categoría $x_{2}$, mientras que los términos $\frac{\beta_{2} \Delta \alpha}{\mu(\delta+\alpha+\mu)(\gamma+\mu+\phi)}+\frac{\gamma \alpha}{(\delta+\alpha+\mu)(\gamma+\mu+\phi)}$ representan los nuevos casos que producen las personas infectadas con tratamiento antirretroviral, los cuales se encuentran en la categoría $x_{3}$.

$\Delta \quad$ Del primer término $\frac{\beta_{1} \Delta}{(\delta+\alpha+\mu) \mu}$ se tiene que $\frac{\Delta}{\mu}$ : población susceptible en ausencia de infección, $\beta_{1}$ representa la tasa de contactos efectivos entre susceptibles e infectados sin tratamiento y $\frac{1}{\delta+\alpha+\mu}$ indica el periodo infeccioso de una persona infectada sin tratamiento antirretroviral.

$$
\text { El segundo término } \frac{\beta_{2} \Delta \alpha}{\mu(\delta+\alpha+\mu)(\gamma+\mu+\phi)} \text {, }
$$
se tiene que $\beta_{2}$ es la tasa de contactos efectivos entre susceptibles e infectados con tratamiento, $\frac{1}{\gamma+\mu+\phi}$ es el periodo infeccioso de una persona infectada con tratamiento antirretroviral y $\alpha$ es la tasa de personas infectadas que acceden al tratamiento antirretroviral. El tercer término $\frac{\gamma \alpha}{(\delta+\alpha+\mu)(\gamma+\mu+\phi)}$ tiene elementos ya interpretados, por lo cual solo se dará la interpretación del restante, este es $\gamma$, el cual indica proporción de personas que abandonan el tratamiento antirretroviral. 


\subsubsection{Puntos de equilibrios}

Los puntos de equilibrio indican soluciones constantes que satisfacen el sistema no lineal, es por esto que se considera $\frac{d x_{1}}{d t}=0, \frac{d x_{2}}{d t}=0, \mathrm{y} \frac{d x_{3}}{d t}=0$, resultando así un sistema algebraico no lineal, que al resolverlo permite determinar los puntos de equi-

librio. El sistema presenta dos puntos de equilibrios:

$$
E_{0}=\left(\frac{\Delta}{\mu}, 0,0\right) \text { y } \quad E_{1}=\left(\bar{x}_{1}, \bar{x}_{2}, \bar{x}_{3}\right)
$$

Donde

$$
\begin{gathered}
\bar{x}_{1}=\frac{(\delta+\alpha+\mu)(\mu+\phi+\gamma)-\gamma \alpha}{\beta_{1}(\gamma+\mu+\phi)}, \quad \bar{x}_{2}=\gamma+\mu+\frac{\phi}{\alpha} \bar{x}_{3} \\
\bar{x}_{3}=\frac{\alpha \mu(\delta+\alpha+\mu)(\gamma+\mu+\phi)\left(R_{0}-1\right)}{\left(\beta_{1}(\gamma+\mu+\phi)+\beta_{2} \alpha\right)[(\delta+\alpha+\mu)(\mu+\phi)+\gamma(\delta+\mu)]}
\end{gathered}
$$

El punto de equilibrio $E_{0}$ indica que el Virus de Inmunodeficiencia humana no se encuentra en la población; es decir, no se encuentran personas infectadas, este punto de equilibrio puede llamarse en ausencia de infección o en ausencia de enfermedad. Por otro lado el punto de equilibrio $E_{1}$ representa que en la población se encuentran personas susceptibles e infectadas con VIH; en particular, el grupo de las personas infectadas con VIH se divide en dos subgrupos, las personas infectadas con VIH sin tratamiento y los infectados con tratamiento.

La estabilidad del punto de equilibrio $E_{0} \mathrm{y}$ del punto de equilibrio $E_{1}$ se encuentran descritas en las siguientes proposiciones:

Proposición 2: El equilibrio libre de infección $E_{0}$ es local y asintóticamente estable si y solo si $R_{0}<1$.

Prueba: Se evalúa la matriz jacobiana en el punto de equilibrio $E_{0}=\left(\frac{\Delta}{\mu}, 0,0\right)$, con lo que se obtiene:

$$
J\left(E_{0}\right)=\left(\begin{array}{ccc}
-\mu & -\frac{\beta_{1} \Delta}{\mu} & -\frac{\beta_{2} \Delta}{\mu} \\
0 & \frac{\beta_{1} \Delta}{\mu}-(\delta+\alpha+\mu) & \frac{\beta_{2} \Delta}{\mu}+\gamma \\
0 & \alpha & -(\mu+\phi+\gamma)
\end{array}\right)
$$

La ecuación característica de la matriz $J\left(E_{0}\right)$ está dada por:

$$
(\lambda+\mu)\left(\lambda^{2}+\alpha_{1} \lambda+\alpha_{2}\right)=0
$$

Donde

$$
\begin{gathered}
a_{1}=\mu+\phi+\gamma+(\mu+\delta+\alpha)\left(1-R_{0}+\frac{\left(\beta_{1} \Delta+\gamma \mu\right) \alpha}{\mu(\delta+\alpha+\mu)(\gamma+\mu+\phi)}\right) \\
\text { y } a_{2}=1-R_{0}
\end{gathered}
$$

Por lo tanto un valor propio es $\lambda_{1}=-\mu$ el cual es siempre negativo, para establecer los signos de la parte real de los otros dos valores propios se acude al criterio de Routh-Hurwitz (Perko, 2013) que permite establecer que cuando $a_{1}>0$ y $a_{2}>0$ entonces la ecuación cuadrática tiene solución con parte real negativa, y esto se cumple cuando $R_{0}<1$.

Así $R_{0}<1$ es condición suficiente y necesaria para que el punto de equilibrio $E_{0}$ sea local y asintóticamente estable.

Proposición 3: El equilibrio en presencia de infección $E_{1}$ es local y asintóticamente estable si y solo si $R_{0}>1$.

Prueba: Se evalúa la matriz jacobiana en el punto de equilibrio $E_{1}=\left(\bar{x}_{1}, \bar{x}_{2}, \bar{x}_{3}\right)$, con lo que se obtiene:

$J\left(E_{1}\right)=\left(\begin{array}{ccc}-\beta_{1} \bar{x}_{2}-\beta_{2} \bar{x}_{3}-\mu & -\beta_{1} \bar{x}_{1} & -\beta_{2} \bar{x}_{1} \\ \beta_{1} \bar{x}_{2}+\beta_{2} \bar{x}_{3} & \beta_{1} \bar{x}_{1}-(\alpha+\delta+\mu) & \beta_{2} \bar{x}_{1}+\gamma \\ 0 & \alpha & -(\gamma+\mu+\phi)\end{array}\right)$

La ecuación característica de la matriz $J\left(E_{1}\right)$ está dada por: 


$$
\lambda^{3}+\alpha_{1} \lambda^{2}+\alpha_{2} \lambda+\alpha_{3}=0
$$

Donde:

$$
\begin{gathered}
a_{1}=\frac{\beta_{1}(\delta+\alpha+\mu)(\gamma+\mu+\phi)}{\beta_{1}(\gamma+\mu+\phi)+\beta_{2} \alpha}+(\delta+\alpha+\mu)+\frac{\beta_{1} \gamma \alpha}{\beta_{1}(\gamma+\mu+\phi)+\beta_{2} \alpha}+ \\
\bar{x}_{2} \beta_{1}+\bar{x}_{3} \beta_{2}+\gamma+2 \mu+\phi \\
a_{2}=(\delta+\alpha+\mu)\left(\bar{x}_{2} \beta_{1}+\bar{x}_{3} \beta_{2}\right)+(\mu+\phi+\gamma)\left(\bar{x}_{2} \beta_{1}+\bar{x}_{3} \beta_{2}+\mu\right)+ \\
\frac{\beta_{2} \alpha \mu(\delta+\alpha+\mu)+\beta_{1} \mu \gamma \alpha}{\beta_{1}(\gamma+\mu+\phi)+\beta_{2} \alpha} \\
a_{3}=[(\delta+\alpha+\mu)(\mu+\phi)+\gamma(\delta+\mu)]\left(\beta_{1} \bar{x}_{2}+\beta_{2} \bar{x}_{3}\right)
\end{gathered}
$$

Se hace evidente que $a_{1}>0, a_{2}>0$ y $a_{3}>0$, y teniendo en cuenta la regla de los signos de descartes se puede afirmar que todas las raíces de la ecuación característica tiene parte real negativa y así $R_{0}>1$ (si $R_{0}>1$ entonces $\bar{x}_{1}>0, \bar{x}_{2}>0$ y $\bar{x}_{3}>0$ ) es condición suficiente y necesaria para que el punto de equilibrio $E_{1}$ sea local y asintóticamente estable.

\subsubsection{Umbral de adherencia en función} del acceso al tratamiento

Teniendo en cuenta la Proposición 2, y considerando $R_{0}$ como una función de los parametros $\alpha$ y $\gamma$, se tiene que la enfermedad es controlada si $R_{0}(\alpha$, $\gamma)<1$. Con el fin de determinar un valor umbral de $\gamma$ en función de $\alpha$ tal que $R_{0}(\alpha, \gamma)<1$, se tiene que $\gamma$ $<g(\alpha)$ donde:

$$
g(\alpha)=\frac{(\mu+\phi)\left[1-R_{0}(\alpha, 0)\right]}{\left(\beta_{1} \Delta+\frac{\mu \alpha}{(\delta+\alpha+\mu) \mu}-1\right)}
$$

o explícitamente

$$
g(\alpha)=\frac{\left(\mu^{2}+\beta_{2} \Delta\right) \alpha}{R_{0}(0,0)-1}+\frac{\mu^{2} \delta+\mu \delta \phi+\mu \alpha \phi+\mu^{3}+\mu^{2} \phi-\beta_{1} \Delta(\mu+\phi)}{\beta_{1} \Delta-\mu \delta-\mu^{2}}
$$

En la Figura 1 se muestra la recta $\gamma=g(\alpha)$, además se ilustra la región $\gamma<g(\alpha)$, dicha región representa un conjunto de valores $(\alpha, \gamma)$ donde propician $R_{0}(\alpha, \gamma)<1$, al igual en la figura se ilustra la región $\gamma>g(\alpha)$, en esta región los valores $(\alpha, \gamma)$ establecen $R_{0}(\alpha, \gamma)>1$. De esta manera, en la Figura 2 se establecen los distintos valores de $\alpha$ y $\gamma$ en los cuales se propicia un escenario para que $R_{0}(\alpha, \gamma)<1, R_{0}(\alpha$, $\gamma)>1$ y $R_{0}(\alpha, \gamma)=1$.

En el Tabla 2 se muestran valores de $\alpha$, los cuales representan el acceso efectivo que se debe garantizar a las personas infectadas con VIH, y a partir de ellos se obtuvo el porcentaje máximo de fracaso terapéutico que se puede presentar en la población infectada, buscando así un $R_{0}(\alpha, \gamma)<1$, lo que sugiere teóricamente y bajo los supuestos de este modelo condiciones de evitabilidad para evitar manifestaciones de la infección.

Figura 1. La gráfica $\gamma=g(a)$, divide el plano, $(a, \gamma)$ en dos regiones, donde se representa de color gris oscuro $R_{0}(a, \gamma)<1$ y en la región de color gris claro se muestra $R_{0}(a, \gamma)>1$, utilizando los valores de los parámetros de la Tabla 1

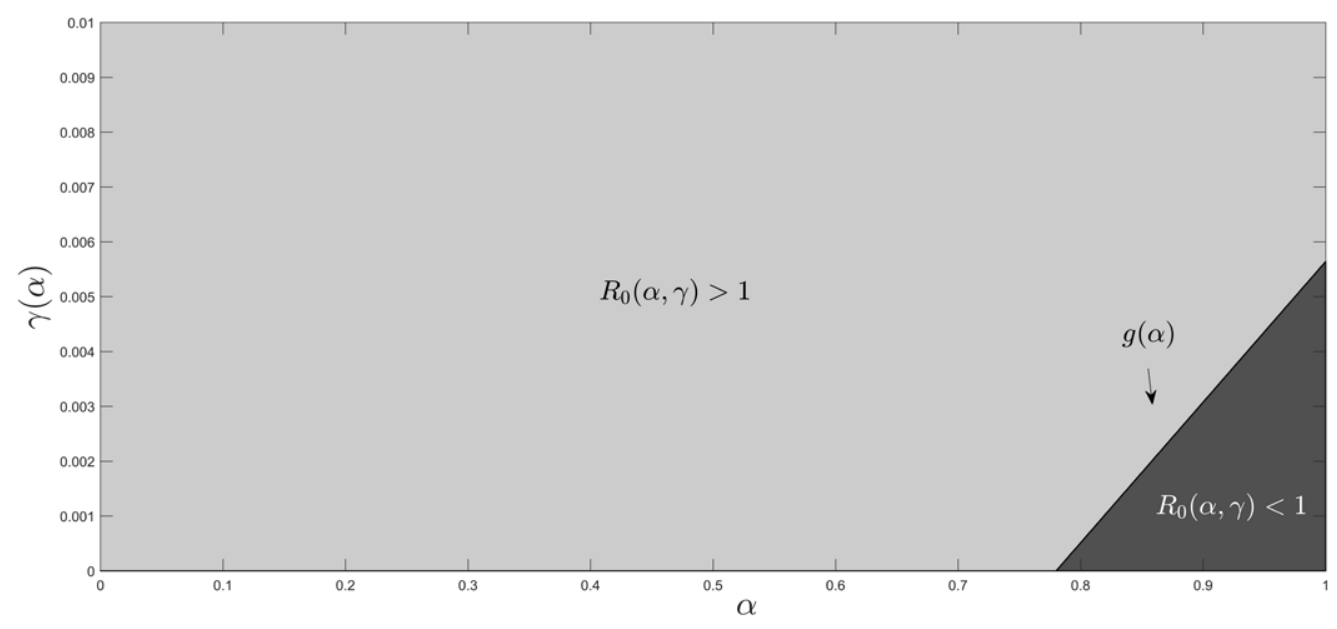


TABLA 2. VALORES DE ACCESO EFECTIVO Y FRACASO TERAPÉUTICO AL TRATAMIENTO ANTIRRETROVIRAL QUE EVITAN TEÓRICAMENTE MANIFESTACIONES DE LA INFECCIÓN.

\begin{tabular}{c|c|}
\hline Valor de $\boldsymbol{a}$ & $\boldsymbol{\gamma}<\boldsymbol{g}(\boldsymbol{a})$ \\
\hline $79 \%$ & $\gamma<0.03 \%$ \\
\hline $80 \%$ & $\gamma<0.05 \%$ \\
\hline $85 \%$ & $\gamma<0.17 \%$ \\
\hline $90 \%$ & $\gamma<0.3 \%$ \\
\hline $95 \%$ & $\gamma<0.4 \%$ \\
\hline $100 \%$ & $\gamma<0.56 \%$ \\
\hline
\end{tabular}

\subsubsection{Umbral de acceso a tratamiento}

Se considera que el abandono a la terapia antirretroviral es nulo, es decir, $\gamma=0$ con el fin de establecer un nivel mínimo de acceso a la terapia antirretroviral, tal que $R_{0}(\alpha, 0)<1$, es así que se tiene:

$$
\alpha>\frac{(\delta+\mu)\left(R_{0}(0,0)-1\right)}{1-H}
$$

Donde $H=\frac{\beta_{2} \Delta}{\mu(\mu+\phi)}$ y $R_{0}(0,0)=\frac{\beta_{1} \Delta}{\mu(\delta+\mu)}$, para que el umbral de acceso tome sentido se debe establecer que $H<1$ y $R_{0}(0,0)>1$; en efecto $R_{0}(0,0)>$ 1 garantiza que la infección se ha establecido en la población, ya que en caso contrario y según la proposición 3 no tiene sentido controlarla.
Por otro lado si $R_{0}(0,0)>1$ entonces se requiere $H<1$ para que el lado derecho de (5) sea no negativo.

\subsection{Simulaciones}

Se realizaron simulaciones del sistema de ecuaciones diferenciales; en las Figuras 2-5 se observa el comportamiento de las personas susceptibles, infectadas sin tratamiento e infectadas con tratamiento teniendo en cuenta los valores para los parámetros que se encuentran en el Tabla 1.

En la Figura 2 se considera una variación en los valores de $\gamma$ en un intervalo de [0,1], en donde la línea (-) representa el valor de $\gamma=0.3$, la línea (- $)$ representa el valor de $\gamma=0.9$, la línea $(-\cdot-)$ representa el valor de $\gamma=0.5$ y la línea $(\cdots)$ representa el valor de $\gamma=0.7$, y con un valor fijo de $\alpha=0.6$, el cual se considera a partir de la Figura 1 garantizando un $R_{0}(\alpha, 0)>1$.

En la Figura 2 estableciendo una variación en los valores de $\gamma$ en un intervalo de [0,1], en donde la línea (-) representa el valor de $\gamma=0.000002$, la línea (- -) representa el valor de $\gamma=0.0015$, la línea $(-\cdot)$ representa el valor de $\gamma=0.0012$ y la línea $(\cdots)$ representa el valor de $\gamma=0.0009$, y con un valor fijo de $\alpha=0.85$, el cual se considera a partir de la Figura 1 garantizando un $R_{0}(\alpha, 0)<1$.

Se realiza una variación en las simulaciones en donde se considera el valor de $\gamma=0$ y variando el valor $\operatorname{de} \alpha$, con lo que se obtiene.

Figura 2. Dinámica de las poblaciones con $R_{0}(a, \gamma)>1$ y para valores de $\gamma=(0.3,0.5,0.7,0.9)$ que corresponden a las curvas $(-,-\cdot-, \cdots,--)$, con los correspondientes valores de $R_{0}(a, \gamma)=(1.58,1.6,1.605,1.608)$
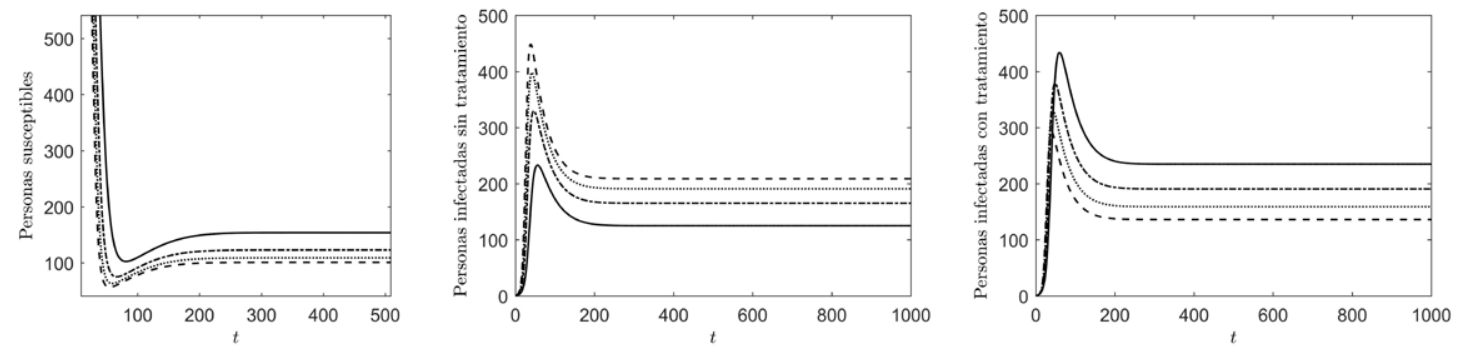
Figura 3: Dinámica de las poblaciones con $R_{0}(a, \gamma)<1$ y para valores de $\gamma=(0.000002,0.0009,0.0012,0.0015)$ que corresponden a las curvas $(-, \cdots,-\cdot-,--)$ con los correspondientes valores de $R_{0}(a, \gamma)=(0.96,0.98,0.98,0.99)$
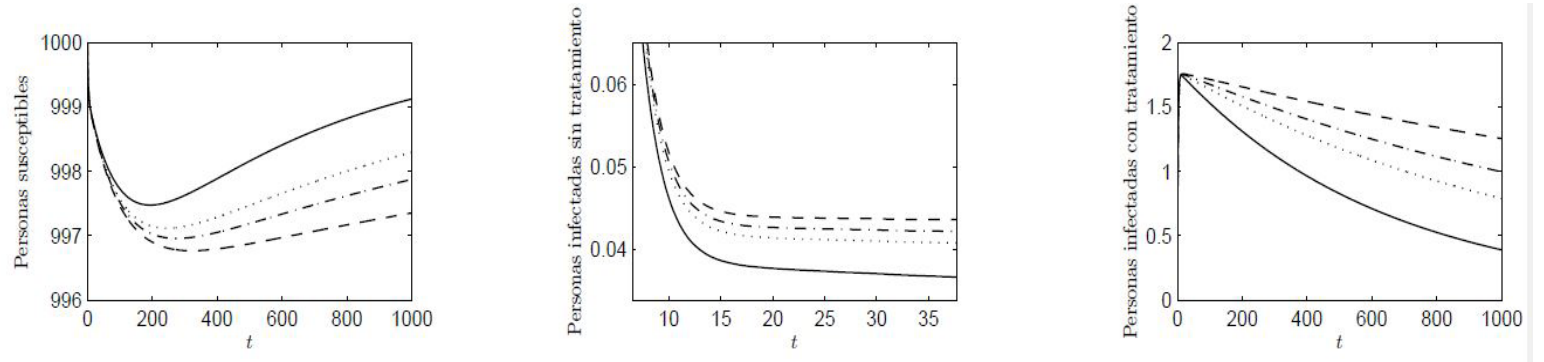

Figura 4. Dinámica de las poblaciones con $R_{0}(a, 0)>1$ y para valores de $a=(0.3,0.45,0.6)$ que corresponden a las curvas $(\cdots,--,-)$ con los correspondientes valores de $R_{0}(a, 0)=(1.72,1.34,1.14)$
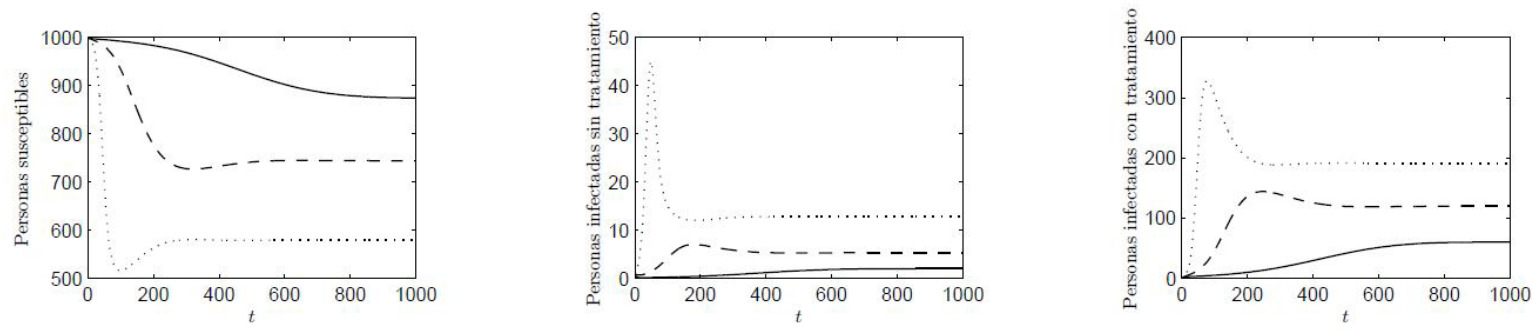

Figura 5. Dinámica de las poblaciones con $R_{0}(a, 0)<1$ y para valores de $a=(0.8,0.88,0.95)$ que corresponden a las curvas $(\cdots,--,-)$, con los correspondientes valores de $R_{0}(a, 0)=(0.98,0.94,0.91)$
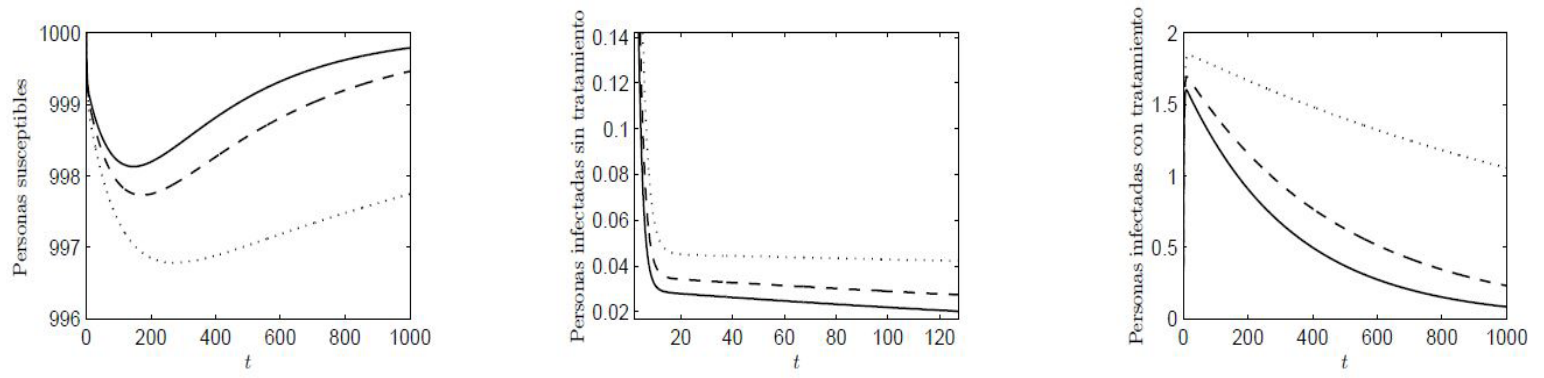

En la Figura 4 se realiza una variación en los valores de $\alpha$ en un intervalo de [0,1], en donde la línea ( $(-)$ representa el valor de $\alpha=0.6$, la línea (- - ) representa el valor de $\alpha=0.45$, y la línea $(\cdots)$ representa el valor de $\alpha=0.3$, los valores de $\alpha$ implementados ilustran un escenario donde $R_{0}(\alpha, 0)>1$.

En la Figura 5 considerando una variación en los valores de $\alpha$ en un intervalo de [0,1], en donde la línea ( - ) representa el valor de $\alpha=0.95$, la línea $(--)$ representa el valor de $\alpha=0.88$, y la línea $(\cdots)$ representa el valor de $\alpha=0.8$, los valores de $\alpha$ implementados ilustran un escenario donde $R_{0}(\alpha, 0)<1$.

\section{Problema de control óptimo}

Considere un funcional de costos que acumula los costos directos e indirectos del acceso efectivo y el fracaso terapéutico al TAR.

$$
J(\alpha(t), \gamma(t))=\int_{\Omega}^{\tau}\left[A x_{2}(t)+\frac{1}{2}\left(B \alpha^{2}(t)+C \gamma^{2}(t)\right)\right] d t
$$


Donde $\alpha(t)$ es la función de acceso efectivo al TAR en función del tiempo $t$ y $\gamma(t)$ es la función de fracaso terapéutico en función del tiempo $t$, ambas funciones definidas en el intervalo $[0, \tau]$, y $A, B$ y $C$ son ponderadores usados para equilibrar las unidades de los términos que se suman en (8).

Considere $\Gamma=\left\{(\alpha(t), \gamma(t)) \in \mathrm{L}^{2}([0, \tau]): 0 \leq \alpha(t)\right.$ $\leq 1,0 \leq \gamma(t) \leq 1\}$ el conjunto de controles admisibles. El objetivo es minimizar $J(\alpha(t), \gamma(t))$ es decir encontrar un par de funciones óptimas $\left(\alpha^{*}(t), \gamma^{*}(t)\right)$ tal que

$J\left(\alpha^{*}(t), \gamma^{*}(t)\right) \leq J(\alpha(t), \gamma(t)) ;$ para todo $(\alpha(t)$, $\gamma(t)) \in \Gamma$

Se aplica el Principio del Máximo de Pontryagin para estudiar el problema de control óptimo, para esto se tiene en cuenta $u(t)=(\alpha(t), \gamma(t))$ y se determina el siguiente Halmitoniano

$$
\begin{aligned}
H(x(t), \lambda(t), u(t))= & A x_{2}+\frac{1}{2}\left(B \alpha^{2}+C \gamma^{2}\right)+\lambda_{1}(\Delta- \\
& \left.\beta_{1} x_{1} x_{2}-\beta_{2} x_{1} x_{3}-\mu x_{1}\right)+\lambda_{2}\left(\beta_{1} x_{1} x_{2}\right. \\
& \left.+\beta_{2} x_{1} x_{3}+\gamma x_{3}-(\delta+\alpha+\mu) x_{2}\right)+\lambda_{3} \\
& \left(\alpha x_{2}-(\mu+\phi+\gamma) x_{3}\right)+z_{1} \alpha+z_{2} \\
& (1-\alpha)+z_{3} \gamma+z_{4}(1-\gamma)
\end{aligned}
$$

donde $\lambda_{i}(t) \in \mathbb{R}$ con $i=1$, 2, 3 son funciones continuas que denotan variables de coestado y los $z_{i}$ con $i=1,2$, 3 son cantidades de penalización positivas usadas para garantizar que $0 \leq \alpha(t) \leq 1$ y $0 \leq$ $\gamma(t) \leq 1$.

Según la función Hamiltoniana tenemos:

$$
H\left(x^{*}(t), \lambda^{*}(t), u^{*}(t)\right)=\min _{u \in \Gamma} H\left(x^{*}(t), \lambda^{*}(t), u^{*}(t)\right)
$$

Aplicando la condición de primer orden $\frac{\partial H}{\partial \alpha}=0$
$\frac{\partial H}{\partial \gamma}=0$, se obtiene: $\alpha^{*}=\min \left\{\frac{\lambda_{2}-\lambda_{3}}{B} x_{2}, 1\right\}$ y $\gamma^{*}=\min \left\{\frac{\lambda_{3}-\lambda_{2}}{C} x_{3}, 1\right\}$
El sistema adjunto se describe a través de las variables $\lambda_{1}, \lambda_{2}$, y $\lambda_{3}$, las cuales están determinadas por medio de la siguiente expresión $\lambda_{1}=-\frac{\partial H}{\partial x_{i}}$ para $i=1,2$, 3. Explícitamente

$$
\begin{aligned}
& \frac{d \lambda_{1}}{d t}=\lambda_{1}\left(\beta_{1} x_{2}+\beta_{2} x_{3}+\mu\right)-\lambda_{2}\left(\beta_{1} x_{2}+\beta_{2} x_{3}\right) \\
& \frac{d \lambda_{2}}{d t}=-A+\lambda_{1} \beta_{1} x_{1}+\lambda_{2}\left(\delta+\alpha+\mu-\beta_{1} x_{1}\right)-\lambda_{3} \alpha \\
& \frac{d \lambda_{3}}{d t}=\lambda_{1} \beta_{2} x_{1}-\lambda_{2}\left(\beta_{2} x_{1}+\gamma\right)+\lambda_{3}(\mu+\phi+\gamma)
\end{aligned}
$$

con las condiciones terminales $\lambda_{1}(\tau)=0, \lambda_{2}(\tau)$ $=0$, y $\lambda_{3}(\tau)=0$. El problema de contorno está dado por el sistema de variables de estado (4) los controles óptimos (9) y el sistema adjunto (10).

\subsection{Resultados numéricos}

Se simula el problema de contorno utilizando el software Matlab, con los valores de los parámetros dados en la Tabla 1, con un valor fijo del ponderador $A=1$ y $C=10$ y variando el ponderador $B$ con valores de 10, 50, 110, y 160.

Se observa a partir de las simulaciones, que, al variar los valores del ponderador $B$, esto es al considerar costos mayores generados por brindar acceso efectivo, se produce una curva óptima que refleja niveles bajos de acceso a los antirretrovirales, caso contrario si se establece un bajo costo de acceso efectivo, la curva óptima generada muestra que se debería garantizar un mayor porcentaje de acceso. Por lo anterior, se podría considerar que la relación entre el costo de acceder de forma efectiva al tratamiento y el porcentaje óptimo que se debe garantizar para brindar control en la población infectada son inversamente proporcionales. También se observa que en los diferentes escenarios propuestos se debe asegurar que el nivel óptimo de adherencia a los antirretrovirales sea del 100\%, para obtener así un escenario donde el VIH tenga un nivel de control adecuado. 
Figura 6. Simulación del problema de contorno formado por (4), (9) y (10), utilizando los valores de los parámetros de la Tabla 1 y con los valores de los ponderados $A=1, C=10$ y $B=(10,50,110,160)$ que corresponden a las curvas $(\cdots,-\cdot-,--,-)$
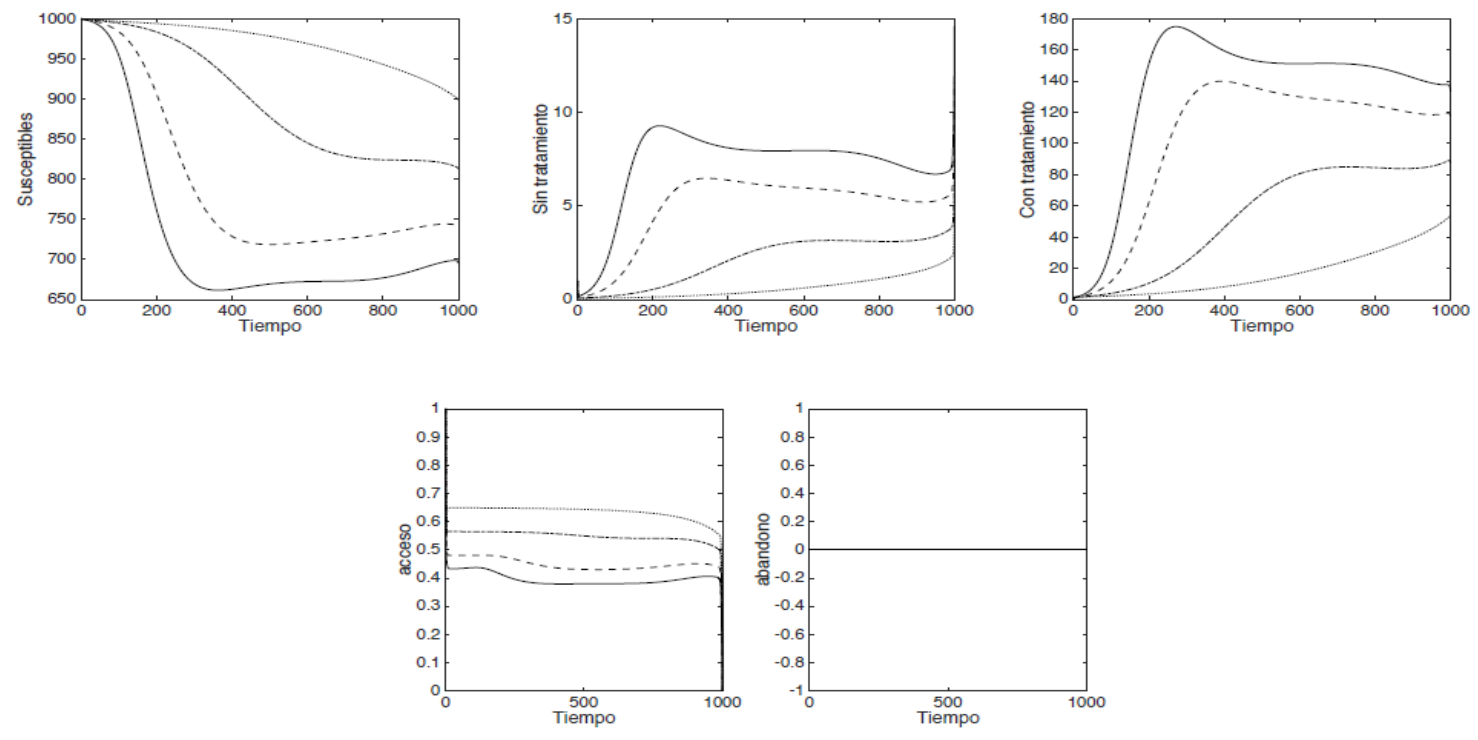

\subsection{Controles subóptimos}

Por medio de la medida de control de acceso efectivo al tratamiento antirretroviral, se pretende disminuir el número de infecciones generadas a causa del VIH, aunque la implementación de esta medida de control se ve afectada por diferentes factores; es por esto que se buscan, estrategias de forma constante y más simples que la función óptima, logrando así que los protocolos de tratamiento dado que no cambian diariamente, se ajusten de manera adecuada o mucho más fácil a la curva de control, estas estrategias las denominaremos estrategias subóptimas.

Control óptimo promedio: una de las estrategias subóptimas consideradas, como medida de control, es el control óptimo promedio, este control se considera con el fin de tener una estrategia no muy lejana a la óptima ya considerada (ver Figura 6) pero con el cual se tiene una medida constante, la cual podría facilitar la utilización del tratamiento antirretroviral; para determinar esta medida de control, se hizo uso del valor promedio de una función y consiste en determinar: $\alpha_{m}^{*}=\frac{1}{\tau} \int_{0}^{\tau} \alpha^{*}(t) d t$ donde $\alpha^{*}(t)$ es la medida de control óptimo

Utilizando los valores de los parámetros de la Tabla 1, el control óptimo promedio es de $\alpha_{m}^{*}=$ 0.419 .

Regresión lineal: se considera otra medida subóptima, por medio de una regresión lineal, generada a partir de los valores tomados en el control óptimo, con la cual se pretende considerar una medida de control de forma variable que propicie valores similares o muy cercanos a los valores de la curva óptima, con el fin de que sea una curva más simple que la función óptima. Para determinar la regresión lineal se utiliza el comando polyfit de Matlab, y esta regresión tiene como ecuación:

$$
\alpha(t)=-0.0001 t+0.7217
$$

en donde se observa que la pendiente de la recta de regresión es negativa con lo que indica que a medida que el tiempo aumente, la tasa de acceso efectivo disminuirá.

Valor máximo: la última estrategia subóptima aquí considerada, se hace por medio del valor máximo del control óptimo, la cual está dada por: 


$$
\alpha_{\max }^{*}=\max _{t \in[0, \tau]}\left\{\alpha^{*}(t)\right\}
$$

utilizando los valores de los parámetros de la Tabla 1, $\alpha_{\max }^{*}=1$.

La Figura 7 ilustra la curva óptima, considerando los valores de los parámetros dados en el Tabla 1 y con un valor de $B=10$, al igual se muestra el valor máximo de la curva óptima, el valor promedio de ella y la regresión lineal.

En la Figura 7 se observa que la dinámica de la línea promedio y de la regresión lineal, varían de forma cercana a la curva óptima, haciendo que ellas se encuentren por encima y por debajo de la misma; es por esto que se podría considerar alguna de estas curvas como sustituta aceptable del control óptimo, al momento de no poder considerar la curva óptima como medida de control.
La Figura 8 ilustra el efecto que tiene las distintas estrategias subóptimas, consideras en la dinámica generada por el VIH en la población humana.

La incidencia que tiene la medida del máximo del control óptimo, sobre la dinámica de las poblaciones, como es de esperarse, genera que solo exista población susceptible y que los niveles de infectados ya sea con tratamiento o sin tratamiento antirretroviral sean nulos, al compararse esta estrategia de control con las curvas óptimas mostradas en la Figura 6, se puede observar que sería de gran ayuda para las diferentes poblaciones, pero al momento de observar qué sucede en la relación con el acceso efectivo al tratamiento antirretroviral, mostrado en la Figura 7 se observa que si muchas veces la implementación de medidas óptimas son difíciles, lo sería mucho más la del máximo de la misma.

Figura 7. Curva óptima de acceso efectivo al tratamiento antirretroviral, considerada con el valor del ponderador $B=10 y$ los valores de los parámetros del Tabla 1., donde la función de la curva de regresión es $a(t)=-0.0001 t+0.7217, a_{m}^{*}=0.419$, $\mathrm{y} a_{\max }^{*}=1$

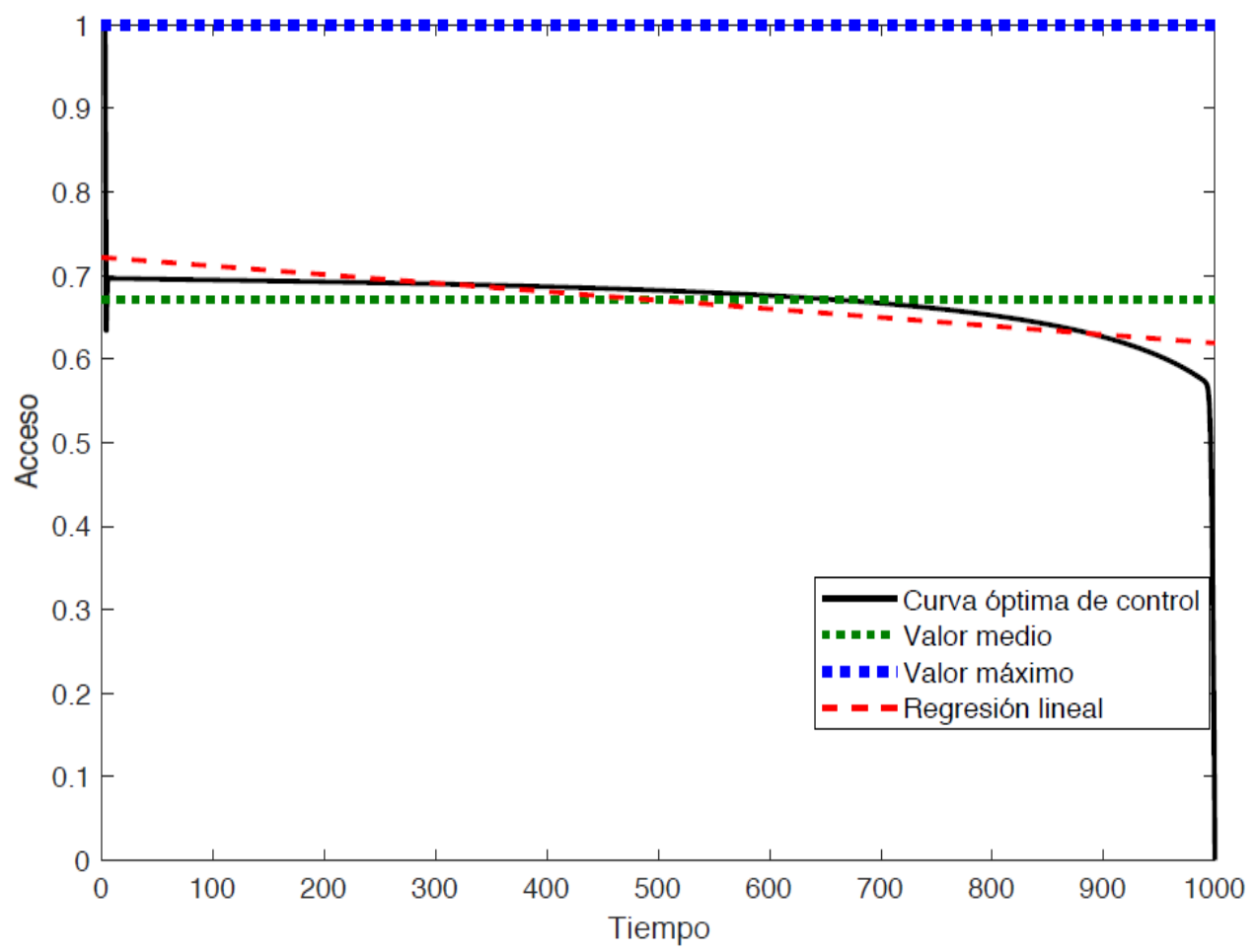


Figura 8. Dinámica de las poblaciones al considerar las distintas estrategias subóptimas
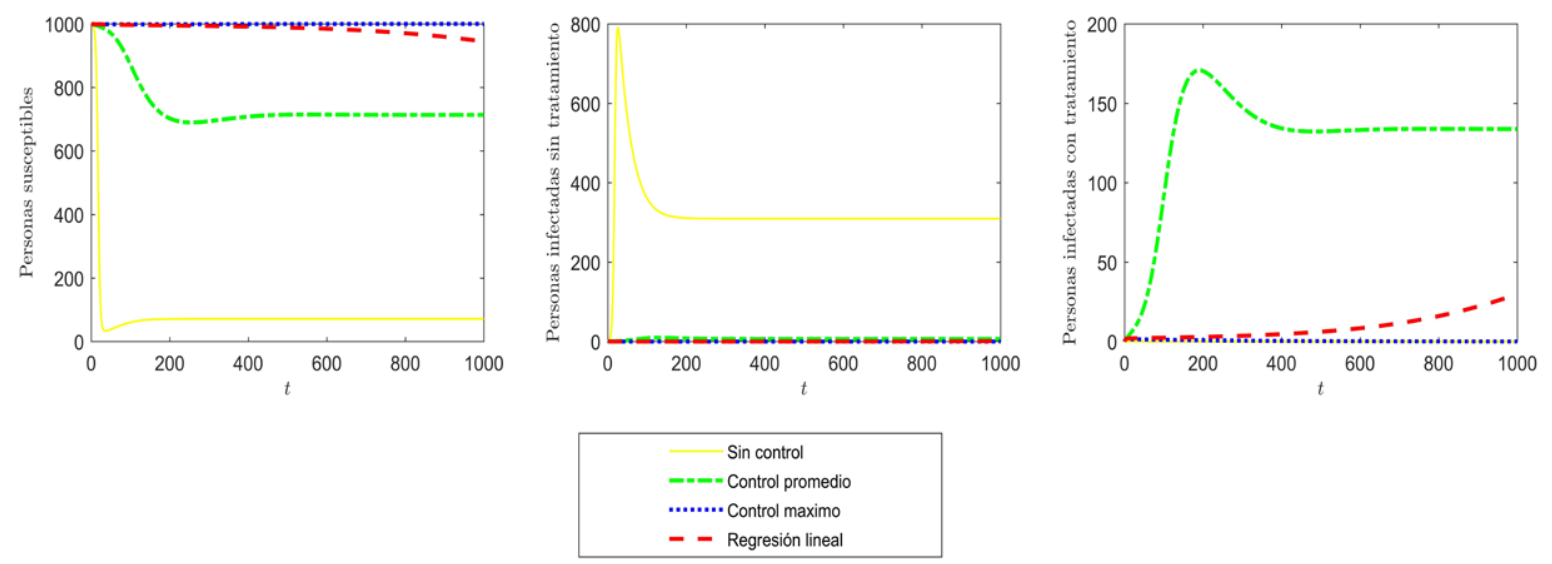

El efecto generado por el control óptimo promedio, evidencia que esta medida de control genera un aumento en la población de susceptibles al igual que la población infectada con tratamiento antirretroviral, y disminuye la población infectada sin acceso efectivo al tratamiento, generando así que la enfermedad sea controlada teóricamente de una manera efectiva evitando que se genere manifestaciones de la infección en la población.

Por último se observa que el control por medio de la regresión lineal, al igual que las otras estrategias subóptimas refleja que la cantidad de infectados sin acceso efectivo al tratamiento antirretroviral tiende a niveles muy bajos, generando así que la enfermedad sin importar la estrategia subóptima utilizada tenga buenos beneficios para la población infectada; si se observa la Figura 6, la cual muestra curvas óptimas, y la Figura 8 se podría afirmar que existe gran similitud en la dinámica de las diferentes poblaciones, por lo cual las medidas subóptimas constituyen una medida de control eficiente.

\section{Conclusiones}

Bajo el modelo planteado, se puede establecer que si el acceso de forma eficaz es limitado (valores bajos de $\alpha$ ), se debe evitar que las personas tengan un fracaso terapéutico (garantizar valores bajos de $\gamma$ ) con el fin de que no se presente manifestaciones de la infección. En contraste, si la adherencia al tratamiento es del $100 \%$, es decir $\gamma=0$, se debe considerar un valor alto de $\alpha$, superior al $70 \%$ con los valores de los parámetros considerados para este caso y los supuestos respectivos, para así garantizar el control de la enfermedad.

En la Tabla 2 se muestran algunos casos especiales en que se determina el valor umbral de $\gamma$ en porcentaje (porcentaje de fracaso terapéutico del tratamiento) que se debe satisfacer, dado el valor de acceso efectivo $\alpha$ en porcentaje. Como puede verse, aún para los niveles más grandes de acceso efectivo, es necesario contar con niveles de fracaso al tratamiento muy bajos. En particular, en el caso hipotético en que se garantiza acceso efectivo al tratamiento antirretroviral al 100\% de la población infectada, se requiere que la tasa de fracaso terapéutico no supere el $0.56 \%$, este escenario se cumple solo se si satisface todos los supuestos del modelo.

En el planteamiento del problema de control óptimo donde se consideran como medidas de control el acceso efectivo y el fracaso terapéutico a la terapia antirretroviral en función del tiempo, para este caso se considera una escala temporal de 1000 días, en el cual se mostró que siempre el nivel de fracaso terapéutico debe ser de cero, mientras que el 
acceso óptimo que se debe brindar varía de forma significativa dependiendo de los valores considerados para los ponderadores, entre más alto se considere el valor del ponderador $B$, más bajos serán los niveles de acceso efectivo al tratamiento antirretroviral, a pesar de estas variaciones en los ponderadores se observó que los niveles de acceso efectivo al tratamiento se encuentran entre el $40 \%$ y el $60 \%$ aproximadamente, todo esto si se cumplen todos los supuestos del modelo.

Según los parámetros usados y el tiempo de simulación considerado, la curva óptima promedio contribuye a que solo se genere población susceptible y población infectada con tratamiento antirretroviral, contribuyendo con un control de manera adecuada. En la medida de control subóptima relacionada con la regresión lineal se ve reflejado que garantiza mejores medidas de control que la curva promedio, dado que disminuye la cantidad de personas infectadas con acceso efectivo al tratamiento, y genera una mayor cantidad de personas susceptibles; a pesar de esto, como es de esperarse, al considerar el valor máximo en la medida de control, la dinámica de las poblaciones no presenta infección, y aunque las simulaciones reflejan que esta sería la mejor manera de controlar la infección se deben considerar factores como el alto costo que esta medida podría demandar, debido a que no corresponde a una solución óptima del problema.

\section{Referencias}

Adams, B. y otros, 2005. HIV dynamics: Modeling, data analysis, and optimal treatment protocols. Journal of Computational and Applied Mathematics, CLXXXIV(1), pp. 10-49.

Campos, U. \& Palacios, E. .. ,. f. d. (., 2005. Análisis y Control de la Dinámica del VIH-1. Journal of Universidad Autónoma de San Luis Potsí.

Chao, D., Davenport, M., Forrest, S. \& Perelson, A., 2004. A stochastic model of cytotoxic T cell responses. Journal of Theoretical Biolog, CCXXVIII(2), pp. 227-240.

Cleary, S., Mooney, G. \& McIntyre, D., 2010. Claims on health care: a decision-making framework for equity, with application to treatment for HIV/AIDS in
South Africa. Health Policy and Planning, XXVI(6), pp. 464-470.

Cuddington, K. \& Beisner, E., 2005. Ecological paradigms lost: routes of theory change. Segunda ed. s.l.:Academic Press.

Culshaw, R., Ruan, S. \& Spiteri, R., 2004. Optimal HIV treatment by maximising immune response. Journal of Mathematical Biology, XLVIII(5), pp. 545-562.

Dalal, N., Greenhalgh, D. \& Mao, X., 2008. A stochastic model for internal HIV dynamics. Journal of Mathematical Analysis and Applications, CCCXLI(2), pp. 1084-1101.

Del Valle, S. M. E. A., Velasco, M., Kribs, C. \& Hsu, S., 2004. Effects of education, vaccination and treatment on HIV transmission in homosexuals with genetic heterogeneity. Mathematical Biosciences, CLXXXVII(2), pp. 111-133.

Ding, A. \& Wu, H., 1999. Relationships between antiviral treatment effects and biphasic viral decay rates in modeling HIV dynamics. Mathematical Biosciences, CLX(1), pp. 63-82.

Gray, R. y otros, 2003. Stochastic simulation of the impact of antiretroviral therapy and HIV vaccines on HIV transmission; Rakai, Uganda. AIDS,. AIDS, XVII(13), pp. 1941-1951.

Grupo Trabajo sobre Tratamientos del VIH, 2011. ¿Cómo se transmite el VIH? [En línea]. Available at: http:// gtt-vih.org/aprende/informacion_basica_sobre_el_ vih/como_se_transmite

Haacker, M., 2002. Modeling the Macroeconomic Impact of HIV/AIDS. s.l.:IMF Working Papers.

Heesterbeek, J., 2002. A Brief History of R0 and a Recipe for its Calculation. Acta Biotheoretic, L(3), pp. 189204.

Hsu, S., 2000. Effects of treatment or/and vaccination on HIV transmission in homosexuals with genetic heterogeneity. Mathematical Biosciences, CLXVII(1), pp. 1-18.

infosida, 2005. Efectos secundarios de los medicamentos contra el VIH. [En línea]. Available at: https://aidsinfo.nih.gov/contentfiles/efectossecundariosdelosmedica

infosida, 2017. ¿Qué es una interacción medicamentosa? [En línea]. Available at: https://infosida.nih.gov/understanding-hiv-aids/fact-sheets/21/95/-que-es-una-interaccion-medicamentosa-

Instituto Nacional deSalud,2016. Boletínepidemiologicosemana 52. [En línea]. Available at: http://www.ins.gov.co/ 
buscador-eventos/BoletinEpidemiologico/2016\%20 Bolet\%C3\%ADn\%20epidemiol\%C3\%B3gico\%20semana\%2052\%20-.pdf

Jiang, D. y otros, 2017. Dynamics of a stochastic HIV1 infection model with logistic growth. Physica A:Statistical Mechanics and its Applications, Volumen CDLXIX, pp. 706-717.

Kouyos, R., Althaus, C. \& Bonhoeffer, S., 2006. Stochastic or deterministic: what is the effective population size of HIV-1?. Trends in Microbiology, XIV(12), pp. 507-511.

Marco, A., 2012. Adherencia al tratamiento antirretroviral e infección por el VIH. Revista Española de Sanidad Penitenciaria, VI(1).

Mesa, M., Zapata, H. \& Prieto, D., 2014. Modelo de simulación para la transmisión del VIH y estrategias de control basadas en diagnóstico. Revista de Salud Pública, XVI(1), pp. 139-152.

Ministerio de Salud y Protección Socia, 2015. Resolución $N^{\circ}$ 5592. Colombia: s.n.

Nelson, P., Murray, J. \& Perelson, A., 2000. A model of HIV1 pathogenesis that includes an intracellular delay. Mathematical Biosciences, CLXIII(2), pp. 201-215.

Oliva, J., López, J., Serrano, P. \& Perestelo, L., 2010. Determinants of health care costs of HIV-positive patients in the Canary Islands, Spain. The European Journal of Health Economics, XI(4), pp. 405-412.

Organización Mundial de la Salud, 2015. Preguntas y respuestas sobre el VIH/SIDA. [En línea]. Available at: http://www.who.int/features/qa/71/es/

Perko, L., 2013. Differential equations and dynamical systems. s.l.:Springer.

Revilla, T. \& García, G., 2003. Fighting a virus with a virus: a dynamic model for HIV-1 therapy. Mathematical Biosciences, CLXXXV(2), pp. 191-203.

Ribeiro, R. \& Bonhoeffer, S., 1999. A stochastic model for primary HIV infection: optimal timing of therapy. AIDS, XIII(3), pp. 351-357.

Santillán, C., 2014. Adherencia terapéutica en personas que viven con VIH/SIDA. [En línea]. Available at: http://132.248.10.225:8080/bitstream/handle/123456789/76/62.pdf?sequence $=1$

Sepulveda, S., Salguero, B. \& Cardona, D., 2011. Dinámica del vih-sida en cali. Revista de Salud Pública, XIII(5), pp. 772-777.

Shim, H., Nam, S., Han, S. \& Seo, J., 2003. Optimal Scheduling of Drug Treatment for HIV Infection: Continuous
Dose Control and Receding Horizon Control. International Journal of Control, Automation and Systems, I(3), pp. 282-288.

Stengel, R., 2008. Mutation and control of the human immunodeficiency virus. Mathematical Biosciences, CCXIII(2), pp. 93-102.

Tan, W. \& Byers, R., 1993. A stochastic model of the HIV epidemic and the HIV infection distribution in a homosexual population. Mathematical Biosciences, CXIII(1), pp. 115-143.

Tarinas, A., Tápanes, R. \& Pérez, L., 2000. Terapia antiviral para VIH-SIDA. Revista Cubana de Farmacia, XXXIII(3), pp. 207-220.

Taylor, G., Cumberland, G. \& P, S., 1994. A Stochastic Model for Analysis of Longitudinal AIDS Data. Journal of the American Statistical Association, LXXXIX(427), pp. 727-736.

Trujillo, C. \& Toro, H., 2015. Simulation Model for AIDS Dynamics and Optimal Control Through Antiviral Treatment. Analysis, Modelling, Optimization, and Numerical Techniques, Volumen CXXI, pp. 257-270.

Varela, T. \& Hoyos, P., 2015. Adherence to treatment for HIV/AIDS: beyond the uptake of antiretrovirals. $R e$ vista de Salud Pública, XXVII(4), pp. 528-540.

Wandeler, G. y otros, 2011. A Comparison of Initial Antiretroviral Therapy in the Swiss HIV Cohort Study and the Recommendations of the International AIDS Society-USA. PLoS ONE, VI(12).

Zaidi, S., 2011. El tratamiento diferencial: Acceso restringido a los tratamientos antirretrovirales más recientes. Crónicas ONU, Volumen XLVIII.

Zaric, G., Bayoumi, A., Brandeau, M. \& Owens, D., 2008. The Cost-Effectiveness of Counseling Strategies to Improve Adherence to Highly Active Antiretroviral Therapy among Men Who Have Sex with Men. Medical Decision Making, XXVIII(3), pp. 359-376.

\section{PARA CITAR ESTE ARTÍCULO / TO REFERENCE THIS ARTICLE / PARA CITAR ESTE ARTIGO /}

Toro-Zapata, H.D.; Osorio-Rodríguez, A.J.; Prieto-Medellin, D.A. (2019). Modelo para el acceso efectivo al tratamiento antirretroviral en relación con el fracaso terapéutico de la infección por VIH. Revista EIA, 16(31), enero-junio, pp. 115130. [Online]. Disponible en: https://doi.org/10.24050/reia. v16i31.1235 